\title{
La participación infantil, una estrategia para la formación del sujeto político en las infancias de Soacha
}

Child participation, a strategy for the formation of the political subject in the childhood of Soacha

Nubia Marcela Gómez*

Alba M. Castro Nemocón ${ }^{* *}$

William A. Prieto Galindo***

Erika Rey Bello****

\section{Resumen}

Niñas y niños presentados como sujetos libres, aportantes en su formación y desarrollo y con ello, en su contexto; son la base para reflexionar sobre la realidad dentro de la cual es pertinente identificar las múltiples posibilidades de participación con las que cuentan y que en ocasiones se limitan, se desconocen, aspectos que se convierten en la problemática del presente artículo, para el que se plantea como propósito presentar las estrategias llevadas a cabo desde el lugar de práctica: Aula móvil, el cual es realizado en algunas comunidades del municipio de Soacha, para generar una propuesta socio educativa que acreciente los niveles de participación infantil y con ello la formación del sujeto político. La investigación se llevó a cabo con el método cualitativo, para el que se establecieron guías de observación, encuestas y entrevistas que permitieron analizar datos acerca de la participación infantil desde el sentir de los

\footnotetext{
* Docente Corporación Universitaria Minuto de Dios, Bogota, Colombia, ngomez6@uniminuto.edu.co http://orcid.org/0000-0002-0714-3114

** Docente Corporación Universitaria Minuto de Dios, Bogota, Colombia, alba.castro@uniminuto.edu http://orcid.org/0000-0001-6312-3583

*** Docente Corporación Universitaria Minuto de Dios, Bogota, Colombia, william.prietog@uniminuto.edu.co http://orcid.org/0000-0001-9988-8238

**** Estudiante Licenciatura en Educación Infantil Corporación Universitaria Minuto de Dios, Bogota, Colombia, erika.rey@uniminuto.edu.co, https://orcid.org/0000-0002-3939-6749
}

\author{
Sinergias educativas \\ Enero - Marzo Vol. 6 - 12021 \\ http://sinergiaseducativas.mx/index.php/revista/ \\ eISSN: 2661-6661 \\ revistasinergia@soyuo.mx \\ Pag 103-123 \\ Recibido: 03 de junio 2020 \\ Aprobado: 02 de diciembre 2020
}

\footnotetext{
Esta obra está bajo una Creative Commons Atribución/Reconocimiento-NoComercialCompartirIgual 4.0 Licencia Pública Internacional CC BY-NC-SA 4.0 https://creativecommons.org/licenses/by-ncsa/4.0/legalcode.es 
adultos, padres o madres de familia, docentes en formación, niñas y niños. Con base en los resultados que se obtienen se fortalece la postura que desde algunas revisiones teóricas se exalta a cerca de la participación de la infancia, la cual se presenta sin relevancia para la población adulta, aspecto tras el cual, resulta de mayor fundamento el trabajo en procura de ésta para favorecer la formación del sujeto político.

Palabras clave: Participación Infantil, sujeto político, infancias, educación

\begin{abstract}
Girls and boys presented as free subjects, contributing to their education and development and thus, in their context; are the basis for reflecting on the reality within which it is pertinent to identify the multiple possibilities of participation with which and that at times are limited, unknown, aspects that become the problem of this article, for the purpose of presenting strategies carried out from the place of practice: Mobile classroom, which is carried out in some communities of the municipality of Soacha, to generate a socio-educational proposal that increases the levels of child participation and thus the training of the political subject. The research was carried out using the qualitative method, for which observation guides, surveys and interviews were established which allowed the analysis of data on child participation from the feelings of adults, parents, teachers in training, girls and boys. Based on the results obtained, the position is strengthened that, since some theoretical reviews, is exalted about the participation of children, which is not relevant to the adult population, after this aspect, the work in search of it to promote the formation of the political subject becomes more fundamental.
\end{abstract}

Key words: Child participation, political subject, childhoods, education.

\title{
Introducción
}

Este artículo acerca a la identificación de los escenarios y las vivencias de las infancias en el municipio de Soacha Cundinamarca, así mismo, aporta en el conocimiento de las diferentes actividades que se presentan en torno a los ejercicios propios de la participación infantil y con ello en el paso del sujeto de derechos al sujeto político.

Es así como, según lo anteriormente expuesto, resulta considerable reconocer el derecho a la participación infantil, el cual es establecido por la Convención sobre los derechos de los niños (CDN, 1989), derecho con el que cuentan y que resulta en ocasiones invisible en los diversos contextos, derecho que ha de reconocer en las infancias la posibilidad de la libertad, en tanto pensamiento, expresión, 
comunicación, religión y conciencia, con el fin de posibilitar a niñas y niños un acercamiento con la realidad que les acontece y de la cual hacen parte, con ello acercar a la población infantil a través de un dinamismo social a aportar en su propia formación y por lo tanto en la transformación de los espacios en los que se vinculas y de los cuales hacen parte (UNICEF, 1990).

El problema de la investigación se concentra alrededor de la carencia de establecer espacios y actividades donde la participación infantil se presente como una estrategia por medio de la cual se aporte en la formación de sujetos políticos activos, comprometidos con aportar en la transformación de sus contextos y con ello en su propio desarrollo, situación que se asevera y fundamenta por medio de estudio de expertos en temas de formación de sujetos partícipes y políticos como Francesco Tonucci (2015), por medio del libro la ciudad de los niños, donde presenta la relevancia del reconocimiento de los niños y la posibilidad que ha de brindársele para el desenvolvimiento y aporte en sus contextos, Ana María Novella Camara (2012), con la investigación La participación infantil: concepto dimensional en pro de la autonomía ciudadana, en el cual fundamenta la participación infantil como el sustento de la formación política y el desenvolvimiento de los niños como líderes en la etapa de la juventud, Bernet Jaume Trillas (2011) con la propuesta de participación, democracia y formación ciudadana: Los consejos de la infancia, con la cual pone en manifiesto la relevancia de la formación infantil en tanto sujetos que participan en la resolución de conflictos, como verdaderos ciudadanos y, Contreras y Perez (2011) con el proyecto, Participación invisible: La niñez y prácticas participativas emergentes, quien presenta las nuevas posibilidades de participación que pueden ser establecidas para niñas y niños, las cuales favorecen su desarrollo social, escolar y familiar.

Con base en dichos autores se logra esclarecer la relevancia de llevar a cabo el presente estudio con el fin de conseguir dar respuesta a ¿De qué manera la participación infantil aporta a la formación del sujeto político en las infancias del municipio de Soacha?

De ésta manera el espacio de práctica itinerante, Aula móvil, de la Licenciatura en Educación infantil de la Corporación Universitaria Minuto de Dios, Uniminuto de Soacha, surge como una estrategia 
dentro de la cual se han vinculado desde 2015 a niños, niñas, padres, madres de familia del municipio y que en este proyecto posibilita el espacio para construir en conjunto una propuesta de participación de niñas y niños entre los 7 y los 11 años, con quienes se fortalezca la formación del sujeto político y con ello aportar en la transformación comunitaria, para ello, se establecen ambientes de socialización, los cuales se encuentran elaborados por estudiantes del semillero de investigación Primera Infancia de la Licenciatura en Educación infantil del Centro Regional Soacha en compañía la docente tutora.

Es importante reconocer que la participación infantil como ejercicio de reconocimiento ha de ubicar a niñas y niños como sujetos que se reconocen y que aportan con postulados que afianzan las construcciones en sus entornos, quienes se han de convertir en la base de los constructos y transformaciones del entorno en el que se hayan, es desde esta perspectiva que Hart, (2013) argumenta sobre la participación infantil que esta conlleva a: "colaborar, aportar y cooperar para el progreso común”, desde ésta perspectiva, aporta en incrementar en los niños, niñas y jóvenes auto confianza e iniciativa personal, ésta aporta en la formación de ellos como sujetos sociales, sujetos con la posibilidad de manifestar sus opiniones de manera espontánea, con un amplio sentido del compromiso, la seriedad en la toma de decisiones a nivel familiar, escolar y social (p.4).

Es así como, el autor presenta una escalera de la participación, por medio de la cual busca ubicar y clarificar los niveles que la componen, para ello, realiza un análisis de la forma en que niñas y niños se vinculan con sus contextos, familiar, comunitario y social, dicho análisis se logra tras un trabajo por proyectos que lleva a cabo el autor, éste parte de la elaboración de un reconocimiento de la situación, a continuación se reflexiona sobre dichas relaciones y por último busca la comprensión de las mismas.

En coherencia con la educación y la forma que desde esta se promueva la participación infantil, cabe aclarar que es una labor que no le atañe únicamente al maestro en el entorno escolar, es una actividad que trasciende a los adultos, al ambiente en el que se encuentra inmerso el niño, para quienes la tarea de orientar, de vincular en el mundo de los cuestionamientos, de la criticidad, de los espacios desde los que niñas y niños logren comprender con mayor 
ahínco el mundo que les rodea, el espacio donde viven, con el fin de destituir acciones puramente políticas que limiten la libertar de pensamiento y generar ambientes dialógicos donde niñas y niños en forma amplia presenten sus posturas ante adultos orientadores, con el fin de elaborar en forma cooperativa elementos que contribuyan en la tranformación y por qué no, generación de una sociedad nueva.

Con base en lo anterior, se resalta la postura de Freire (1997) quien resalta que dentro de los espacios de reflexión se deben posibilitar ejercicios en el que se presente el diálogo y la investigación educativa, las cuales han de convertirse en una forma de participación y evaluación, sin dejar de lado la educación como sustento del anterior, dichas actividades redundan en postulados de poder, criticidad y participación infantil, lo anterior en la medida en que los sujetos al ser parte de espacios educativos, trasforman su realidad, su mirada, su vivir.

Entonces aparece el interrogante, ¿Qué son las infancias? Para dar respuesta al planteamiento, se aborda a Kohan (2020) quien argumenta que existe una relación intrínseca entre tiempo e infancia, en la medida en que con el primero se obtiene la experiencia y con la segunda se vive, entonces aparecen etapas cronológicas para poderla definir y la vida como espacio para recorrer y experimentar, así mismo, Gaitán (2006) la define como "una condición social delimitada por una construcción cultural e histórica diferenciada y caracterizada por relaciones de poder (...) las niñas y los niños serían el grupo de personas o sujetos sociales que se desenvuelven en un espacio social” (p. 83).

Con la convención de los derechos de los niños de 1989 aparece un nuevo postulado en torno al reconocimiento de éstas el cual se fundamenta en los derechos humanos, ésta convención es el primer tratado internacional de derechos humanos que combina en un elemento una serie de parámetros en relación con los niños, a través del cual se establecen los derechos de niñas y niños.

En relación a lo anteriormente expuesto, resulta interesante reflexionar en torno al rol en el que se desempeñan niñas y niños, el cual debe distanciarse del papel de sujetos sumisos, a quienes se les delega el papel de la ternura, espontaneidad, ocurrencia e ingenuidad 
por medio de la cual logran generar distracción para un grupo de seres adultos quienes se consideran sabedores y conocedores del mundo, motivo por el cual se distancian de las contradicciones, de las nuevas posturas y de otorgar el saber a otros inexpertos niños.

Entonces, resulta pertinente agregar a esta conceptualización que la transformación comunitaria ha de ser pensada como una propuesta colectiva, no individual, desde esta perspectiva aparece Escobar (2012), quien argumenta que "el mundo se encuentra lleno de otros mundos" (p. 182), aspectos que permiten llegar al reconocimiento del "otro" como ser humano, como individuo, como sujeto que hace parte de un espacio en el que participa, vive, reacciona desde las propias realidades, pensamientos, sentimiento, intereses y necesidades, en procura de generar propuestas que permitan conseguir la transformación y con ella otra posibles formas de vida.

En coherencia con lo expuesto, y para brindar un hilo conductor con la formación del sujeto político, resulta relevante identificar que la formación de este, se logra por medio de actividades relacionadas con la convivencia social, la participación y los aspectos de aceptación y atención a la individualidad en el espacio social del que hacen parte; en ese caso, aporta a la formación de las propias ideas políticas, que se fundamentan en la expresión del sujeto, en tanto ser político, de allí parte la necesidad del concepto de ciudadanía, del cual, según Peña, (2017) manifiesta: "Lo político es lo constituido desde acciones que implican tomar postura respecto a lo público, es decir, a aquello que nos es común, es entonces al tomar una postura como se configura el sujeto político" (p. 234). Cabe resaltar la importancia del desarrollo de éste sujeto en infancia, involucrando factores de desarrollo integral del niño, desde donde se genera de una manera más amplia, espontánea y eficaz, otro aspecto a resaltar y que fortalece la importancia de la formación del sujeto político en la infancia es la forma en la que se vinculan y llevan a cabo los procesos de ciudadanía por medio de agentes de socialización primarios (Familia y escuela) con los cuales los niños tiene mayor acercamiento, relación y por ende vínculo afectivo, que garantiza una mayor asertividad y construcción integra, dando paso a un desarrollo más completo relacionado con la adaptación, reconocimiento propio, relaciones interpersonales y participación. 


\section{Materiales y métodos}

La propuesta investigativa se lleva a cabo bajo un enfoque cualitativo, el cual permite percibir al ser humano en su contexto desde una perspectiva holística. En este caso, a niñas y niños entre los siete y once años, que se encuentran vinculados en diferentes contextos, en este sentido, tal y como lo define Sandín (2003), "la investigación cualitativa, una actividad sistemática orientada a la comprensión de fenómenos educativos y sociales, a la transformación de prácticas y escenarios socioeducativos, a la toma de decisiones, el descubrimiento y desarrollo de un cuerpo organizado de conocimientos" (p. 123).

Dicha actividad permite llegar a la observación participante, la cual posibilita el análisis de datos y actividades que se llevan a cabo en el escenario de investigación, con base en las cuales se logra detallar, la relevancia que se debe brindar al reconocimiento de los imaginarios, ideas y planteamientos brindados por niñas, niños, padres, madres de familia, docentes en formación que se encuentran vinculados en éstos y fueron quienes facilitaron dicha reflexión y análisis, lo anterior, con base no sólo en posturas, también es el resultado de los ejercicios, talleres y actividades realizadas.

Cabe mencionar que dentro del enfoque en mención se posibilitaron espacios de obtención, reflexión, planteamiento y análisis de datos para los cuales se utilizaron encuestas y entrevistas no estructuradas, así mismo, dichos apartados fueron abordados como fases de la investigación, el diseño de la investigación es la Investigación acción participativa, la cual parte de un ejercicio de observación que es seguido por uno de vinculación del investigador en el contexto propio de la población o campo de trabajo, con el fin de identificar la problemática y con base en ella postular la estrategia para conseguir la resolución de éste.

El paradigma de investigación fue el interpretativo fenomenológico con base en el cual se logra observar la situación del contexto, de los sujetos participantes en la investigación, se reflexiona sobre el mismo y se plantea una posible solución o transformación para éste, en procura de posibilitar un significado nuevo y trascendental para los sujetos participantes del proyecto, en especial el niño como sujeto político. 
Es relevante mencionar que el primer paso de la investigación se logra gracias a la vinculación de la investigadora y los jóvenes en formación que se encuentran vinculados al semillero de investigación Primera Infancia de la Licenciatura en Educación Infantil de la Corporación universitaria Minuto de Dios, Uniminuto, en esta oportunidad se escoge como población de trabajo, la comuna tres del municipio de Soacha, población con la cual se logra un proceso de sensibilización en tanto encuentros periódicos $\mathrm{y}$ actividades semanales que inician con la vinculación a la cotidianidad, las relaciones entre niños, niñas y adultos se generar y como resultado de éstas, se indaga sobre las situaciones que para ellos se convierten en problema, frente a las cuales se escucha de los infantes propuesta de solución y cómo se involucrarían en éstas.

De acuerdo a lo anterior, es por los niños, las niñas, madres y padres de familia, docentes en formación que se genera una propuesta para la cual se construyen e implementan talleres socio educativos a través de los cuales se vinculan los sujetos participantes de las encuestas y entrevistas, quienes se convierten en la base fundamental para el reconocimiento de la realidad, del contexto, se posibilita que los niños sean quienes intenten resolverlas, que sean ellos quienes plantean las alternativas o soluciones, posterior a ello se socializan con los adultos y en conjunto reflexionan sobre los postulados expuestos, se encuentra que coinciden en apreciaciones y presentan alternativas en conjunto para aportar al mejoramiento de la calidad de vida, de la realidad de todos y de la educación.

Dentro de los talleres se tuvieron en cuenta cuatro actividades que fueron fundamentadas con base en la propuesta realizada por la Secretaria de Educación Distrital (2015), A participar aprendo participando, a partir de la cual se lleva a cabo una serie de dinámicas que generan espacios de reflexión, participación infantil y con la cual se buscó fundamentar las actividades propias de los gobiernos escolares, estas fueron adaptadas a la investigación y aportaron en los encuentros con la comunidad de trabajo.

Los talleres implementados fueron el resultado de la revisión teórica y adaptación al contexto, es así como Gomez, (2019) plantea cuatro talleres con la población sujeto de la investigación, éstos son: Reconocimiento de la población y el contexto, en éste primer taller, 
niñas y niños realizan un esquema del entorno en donde se lleva a cabo la actividad, por medio del cual reconocen lugares de mayor importancia para ellos e identifican cuales son las debilidades y fortalezas de dichos espacios, frente a ello postulan oportunidades de mejora para la sana convivencia, en el segundo taller, Las problemáticas del sector niñas y niños reconocen los problemas que se presentan con mayor frecuencia en el contexto, se lleva a cabo una reunión tipo junta comunal, cada uno asume el rol de uno de los líderes de ésta, se les explican dichas tareas, como resultado, se encuentran alternativas formuladas por niñas y niños donde abordan aspectos como la salud, el ambiente, la educación, el deporte, entre otros, algunas posturas sobre cómo minimizar dichas problemáticas, posteriormente se socializa con los madres y padres de familia, docentes en formación, se encuentran coincidencias de las reflexiones de los diferentes miembros de la población participante en la actividad; en el tercer taller, De viaje por el contexto, por medio de un conversatorio grupal, los participantes manifiestan qué identifican de la zona, situación de la cual, se recopilan datos acerca del sentir, pensar y vivir de ellos en torno al contexto en el que se encuentran inmersos, con base en ello, se logra inferir que el reconocimiento, sentimiento y mirada de los infantes en torno al espacio que habitan es una recreación de aspectos transferidos por los mayores, de ésta forma, con la población vinculada en éste taller se lleva a cabo una pequeña reflexión en torno a cómo son los procesos de participación de cada uno de ellos en su contexto, aspectos de los cuales emerge la idea en torno a la participación que cada uno desea y logra tener en éste. En el taller número cuatro, Nuestra Cotidianidad, por medio de actividades pedagógicas, los sujetos participantes en el encuentro permiten observar cómo es el ejercicio de participación en la comunidad, aquí, cada participante presenta cómo se refleja e identifica en su comunidad, lo anterior en torno a la reflexión de cómo desde la realidad personal se participa en la transformación del contexto, para finalizar, se establecen algunas responsabilidades para los adultos, las que se presentan en torno al trabajo y la relación con los niños, estas se encaminan en torno al reconocimiento la escucha, el observar sus posturas, creer en sus ideas y brindarles la opción de participar. 


\section{Resultados}

Para llegar a los resultados de la investigación, se tienen en cuenta tres perspectivas relevantes que fueron aplicadas para la recopilación de la información, inicialmente los resultados de las encuestas, en seguida los análisis de las entrevistas y las reflexiones de los talleres que se logran realizar con la colaboración de la población participante en el estudio. Instrumentos que seguidos de la observación participante, la cual es utilizada en forma secuencial en cada encuentro que se llevó a cabo en el sitio de práctica pedagógica itinerante Aula móvil, sitio de práctica que hace parte de la Licenciatura en Educación Infantil de la Corporación universitaria minuto de Dios, Uniminuto, del Centro Regional Soacha.

Frente a las encuestas, resulta pertinente mencionar que se elaboran dos encuestas mixtas, una para los adultos asistentes y vinculados en el proyecto (madres, padres y docentes en formación) y otra para niñas y niños entre los siete y los once años, con base en las cuales se logran identificar en qué nivel de participación se encuentran ubicados los niños, reflexión para la que se cuenta con la escalera de la participación de Hart (2012), la muestra se toma con 45 encuestas a niñas y niños y 37 a adultos, cada una de éstas cuenta con trece preguntas.

Dentro de los análisis se observa que según Hart (2012), la participación infantil encontrada en el Municipio de Soacha es abordada como una no participación, lo anterior debido a que se ubica en las tres primeras espacios de la escalera de la participación, los cuales son conceptualizados por el autor como espacios de manipulación dentro de los que la comprensión hacia los niños es escasa frente al resultado que obtienen de sus acciones; mientras tanto, aparecen otras apreciaciones que se ubican en el siguiente espacio de la escalera, el cual, el autor le denomina peldaño de la decoración, en éste, los adultos hacen uso de los aportes, ideas y postulados infantiles para afianzar las causas de diferentes temas, hechos o acontecimientos de manera indirecta, aquí, aparece una participación simbólica en la que los infantes cuentan con una aparente oportunidad para expresarse y opinar frente a dichos temas, sin embargo, la realidad conlleva a encontrar que la incidencia de los postulados expuestos por ellos es escasa o nula en la medida en que no cuentan con la oportunidad para formular opiniones propias y 
mucho menos pensar en que éstas se lleguen a ejecutar, situaciones que incrementan el interés por dar continuidad al proceso investigativo y con el poder generar un impacto en diferentes comunas del municipio de Soacha.

Para la realización de la entrevista no estructurada, inicialmente se construye una guía para instaurar los temas que orientan la indagación y con ella recolectar la información de la población participante en torno a la participación infantil y la formación de los niños en los diferentes escenarios donde se vinculan, siendo estos la familia, la escuela, los juegos, la sociedad, entre otros; el esquema que se utiliza posibilita la vinculación de preguntas nuevas, en la medida en que se establece el diálogo con el entrevistado, algunas de estas preguntan generan flexibilidad para que el entrevistado brindara detalles que se convierten en fuentes valiosas para el posterior análisis, en total se llevan a cabo 18 entrevistas a la población participante del proyecto.

Dentro de los análisis a los que se llega con la entrevista se encuentra que los madres y padres de familia, oscilan en el momento de la ésta entre los 36 y 42 años, son residentes del municipio de Soacha Cundinamarca, laboran en diferentes actividades del sector productivo del municipio, con ellos se dialoga sobre la participación infantil, la educación infantil y la transformación comunitaria, sobre la participación infantil se encuentra que de acuerdo a la escala de Hart, (2012), no se cuenta con un ejercicio de ésta, en la medida que la acción de participación de parte de niñas y niños no se lleva a cabo desde la autonomía de ellos, así mismo, no da respuesta a los intereses que desde ellos nacen, hecho por el cual como es expresado por Borda Luz (39 años), las decisiones en casa casi siempre son tomadas por los adultos, en tanto los niños inician participando $\mathrm{y}$ finalizan peleando, así mismo, Luz Dary de 40 años plantea que, para participar se debe contar con varias personas en torno a la cimentación de una idea o de una situación, en esta medida se aborda la convención de los derechos de los niños de 1990, la cual asevera el derecho a la participación que tienen niñas, niños y adolescentes, que en la realidad se deja de lado, no se aborda como un derecho fundamental.

En tanto, los docentes toman una postura específica en la entrevista, 
en ocasiones, ellos relacionan la importancia de la participación infantil con el artículo 12 de la Convención de los derechos de los niños, en el cual se presenta como uno de los cuatro principios fundamentales aportantes al desarrollo infantil, así mismo, tienen en cuenta el derecho a expresarse frente a situaciones que se presenten $\mathrm{y}$ les afecten, argumentan que dicha opinión debe ser tenida en cuenta planteando una relación entre esta, la edad y madurez infantil, otros docentes la abordan como una posibilidad que se brinda a la infancia para generar aportes $y$ discusiones frente a temas específicos que se presentan y de los cuales deben ser escuchados, manifiestan que la participación infantil se relaciona en forma estrecha con la autonomía, aparecen posturas en torno a que se logra la participación con la orientación de los adultos, que los niños deben encontrarse prestos a seguir normas, las cuales han sido dadas por los adultos, aspectos que se relacionan en forma estrecha con la participación simbólica planteada por Hart, (2012) en la escalera de la participación.

Para encontrarse con la educación se resalta la postura de Vela Yolanda (42 años) quien manifiesta la importancia de la educación ya que es por ella que se logra ayudar a los demás, sin interesar el nivel social o económico que cada uno tenga, por lo tanto, aparecen postulados tales como el de Freire (1997) quien manifiesta la importancia de direccionar a niñas y niños hacia una formación como sujetos políticos y sociales con base en la criticidad, a quienes se les posibilite plantear cuestionamientos a través de los cuales logren una interpretación del mundo, de la realidad, del ambiente en el que se vinculan de una forma amplia y detallada, lo anterior, sin olvidar el elemento dialógico en los que se han de vincular para conseguir la transformación de las realidades sociales y políticas en las que se encuentran inmersos, situaciones que según el autor, no solo se posibilitan en el entorno escolar, es más, nacen del contexto familiar.

Los docentes entrevistados, en torno a la educación manifiestan existe con el fin de ayudar a los niños a desarrollar sus capacidades, que gracias a ésta, logran ser eficientes para los sistemas, es considerada la base de la sociedad, argumentan se encuentra permeada por valores y principios que se establecen en casa, en la familia y se corroboran en la escuela 
En cuanto a la concepción que los adultos tienen sobre la infancia se aborda el planteamiento realizado por la madre de familia, Burgos L. (40 años) quien argumenta desde su perspectiva, la etapa infantil es poco reconocida, aspecto que se da debido al olvido de la propia infancia en cada sujeto adulto, al olvido de la educación que se obtuvo durante la vida, aspectos que redundan en no tener en cuenta lo que dicen y piensan los niños, así mismo, plantea que en oportunidades como madre desea que sus hijos actúen como ella, sin tener en cuenta el sentimiento y deseo de los niños, que evidencia que en la comunidad no resulta importante el pensamiento infantil.

Con base en lo anteriormente expuesto, resulta corroborante el planteamiento en torno a la participación de la infancia, la cual se encuentra ligada a la obediencia, a acatar y seguir normas más que a la toma y aporte en las decisiones, desde esta perspectiva, niñas y niños continúan vinculados al sometimiento sin la posibilidad de cuestionar, al silencio y la obediencia, situación que imposibilita la formación del sujeto crítico, político, reflexivo y espontaneo, para ratificar la postura se tiene en cuenta el planteamiento expuesto por Camara, (2012) quien manifiesta que sólo se logra la transformación de las realidades como un resultado de la comprensión colaborativa en y para el mundo, dicha comprensión ha da iniciarse desde la infancia, etapa desde la cual se debe permitir el planteamiento de posturas propias, reforzar la libertad de pensamiento, favorecer espacios de opinión, acontecimientos que se deben potenciar y fortalecer inicialmente desde el vínculo familiar, el cual ha de ser seguido en la escuela y por último, se verá explícito en la sociedad o contexto donde niñas y niños se involucren, dichas actividades aportan y fortalecen la adquisición de la autonomía, la cual se presenta en torno a la formación del sujeto político y la juvenil.

En cuanto a la infancia, los docentes reconocen que la exaltación de esta es superficial, desde ésta perspectiva, no se distancia del planteamiento realizado por Wasseman (2016) a cerca de la infancia, quien la presenta desde el concepto etimológico como aquel sujeto que quien no se le permite hablar, en tanto no cuenta con la legitimidad de la palabra, es así como algunos docentes manifiestan la evidencia de no encontrar a los niños como una prioridad en el entorno en el que se desenvuelven, en ocasiones los convierten en seres que brindan compañía familiar, otros presentan preocupación 
sobre la importancia de hacer respetar y reconocer los derechos de niñas y niños, identificar sus necesidades e intereses, aportar en sus fortalezas e intentar planteamiento de oportunidades frente a sus debilidades, otros, presentan la infancia como el sustento social para lograr el desarrollo, el cambio y la transformación futura.

Tras abordar la transformación comunitaria se encuentran reflexiones en torno a que esta se debe potenciar desde el ser y actuar de cada individuo desde la infancia, así mismo, los docentes plantean que para conseguirla, se debe contar con el aporte de entidades gubernamentales, situación que en ocasión se obstaculiza en espacial por manejos de carácter económico, así mismo, exaltan que a niñas y niños se les debe formar como sujetos reflexivos, críticos, políticos, que para ello, es necesario enseñarles a cuestionar, se les debe orientar, aspectos que deben aparecer desde la primera infancia, con ello se pueden lograr modificaciones significativas para las infancias y para el contexto, con base en lo anterior se retoma la idea Freire (1993), quien plantea que dichos aspectos necesitan que los procesos educativos se presenten como dinámicas de la aprehensión de la criticidad, del cuestionamiento a cerca de las realidades del mundo, que sólo por medio de éstas se logra un acercamiento a la libertad, al poder, aspectos que redundan en la transformación de la realidad.

Las niñas y los niños participantes en la entrevista se encuentran entre los 7 y los 11 años de edad, residen en el municipio de Soacha, manifiestan que participar se asocia con compartir, realizar una actividad que les guste, dentro de lo que se encuentran los juegos o actividades escolares, en tanto, participación en casa mencionan no se les reconoce tal como lo expresa Santamaría Nicolle (8 años) quien comenta su participación en casa se limita, en tanto, considera, las opiniones brindadas por ella en ocasiones son relevantes en el colegio, mientras tanto para Vega Luisa de 12 años, su participación se limita a las preguntas que le planteen directamente, en tanto, reconocen que pueden presentar opiniones para escoger juegos y organizarlos, así como al opinar sobre las actividades a realizar o lugares a visitar en casa, que en oportunidades no se les presta atención o se les tiene en cuenta.

Niños y niñas le dan importancia a la educación, manifiestan que sin 
ella no logran ser mejores seres humanos, la plantean como importante pues les sirve para estudiar, conseguir realización, "ser alguien en la vida", Vega (2018) o para simpatizar a los demás como es mencionado por Arcila de 8 años quien en torno a ésta plantea que le sirve pues como resultado de ésta, logra afecto de los demás y su objetivo es llegar a ser una gran persona, de esa manera, otros de los niños dentro de sus aportes manifiestan preocupación y la consideración que tiene por el desmejoramiento de la educación.

Mientras tanto al hablar de infancia mencionan que para los adultos esta etapa se ha dejado de lado, así lo manifiesta Daniela de 8 años al referir, los adultos olvidaron la infancia, sólo por lo que són, así mismo plantea que la infancia es la mejor etapa, en ésta se puede compartir, adquirir conocimientos, incluso muchos más que los obtenidos por los adultos, reflexiones que se asocian con los postulados de Gaitán, (2006) quien plantea que la infancia es delimitada por construcciones culturales, históricas, sociales, que se encuentran al margen de relaciones de poder, en tanto, los niños plantean que el reconocimiento de ellos es escaso y piden sus decisiones sean tenidas en cuenta y las decisiones sociales se tomen pensando también en los niños y en el bien común.

Entonces cabe mencionar que la reflexión que se tiene desde la infancia en torno a la participación y la formación del sujeto político y crítico no sólo se orienta y fundamenta en su bienestar, trasciende la etapa de la infancia, se proyectan al bienestar en general, en ocasiones dejan de sentir, de pensar, de crear, de imaginar por aportar, por laborar o por desempeñarse en alguna actividad que le aporte a sus familias.

Sobre la transformación comunitaria, para los niños se establecen nuevos interrogantes, en la medida en que según ellos es indescriptible lo que puede acontecer y cómo se pueden vincular, así mismo, la presentan como una oportunidad para que los adultos los identifiquen, los reconozcan, para que las costumbres que tienen los adultos se modifiquen y con ellas la prioridad de escuchar y percibir sentimientos pensamientos, así mismo, manifiestan que encontrarse en la infancia, no es un impedimento para poder aportar y reflexionar sobre el beneficio de todos. 
Desde estas perspectivas se encuentra que a modo general tanto de niños, niñas, madres y padres de familia como de docentes, se corrobora que la participación infantil no se encuentra, desde esta perspectiva, el reconocimiento por la infancia en el municipio de Soacha se limita.

\section{Discusión}

Los procesos de articulación entre la docencia y la investigación, posibilitan el identificar dificultades o problemáticas en diferentes espacios o comunidades, así como generar propuestas que conlleven a plantear y lograr transformaciones, apuestas que han de pensarse desde los procesos de calidad y pertinencia en procura de la posible atención a la población que se identifica como vulnerable.

La Participación infantil como estrategia para la formación del sujeto político, se presenta y genera un aporte en la transformación comunitaria, situación que ha de favorecer la transición de la infancia en tanto sujetos de derechos a sujetos políticos, a través de un fundamento que inicia realizando una revisión epistemológica, para adentrarse a un trabajo pedagógico social, en el que los ejercicios propios de la enseñanza aprendizaje son utilizados en procura de dar respuesta a la problemática evidenciada en un contexto determinado y con una población específica, con el fin de que el estudio se torne concreto y pertinente.

Es necesario generar prácticas desde la participación en las familias, por medio de las cuales, se posibilite a la población infantil generar aportes respecto a situaciones de relevancia familiar, que trasciendan los límites del hogar y aporten a los constructos sociales, que les posibilite a niñas y niños una formación con base en la autonomía, el respeto, el auto concepto, que les enriquezca como seres humanos activos y participantes en una comunidad.

Las dinámicas participativas para las niñas y niños deben ser repensadas, en procura de garantizar procesos de enseñanza aprendizaje que trasciendan del direccionamiento, la enseñanza y el acatar normas, se debe pensar en ejercicios de socialización, escucha, investigación, construcción, donde cada miembro de la sociedad, desde su saber, sentir, pensar y actuar, aporte en la 
transformación del contexto, bases que en este estudio se convierten en primordiales para la formación del sujeto político, así mismo, es pertinente continuar trabajando en ellas.

Desde estas perspectivas se convierte en una de las responsabilidades sociales de la educación el reconocer la participación de niñas y niños, con base en ella, identificar el fin de sus aportes, a los que pueden dar utilidad para conseguir las transformaciones comunitarias, sin embargo, se observa la participación como un ejercicio de no participación, que se encuentra manipulada por los adultos, reflexión a la que se llega luego de las encuestas y entrevistas realizadas y tras la revisión teórica presentada por Hart (2012).

Con la implementación de las técnicas e instrumentos de recolección de la información se obtiene la impresión sobre el significado de la participación infantil, en procura de la formación del sujeto político, de ésta se logra reconocer que para la población adulta resalta que entre más obedientes sean los niños, menores esfuerzos para ellos.

Los participantes de la investigación reflexionan en torno a las diversas posibilidades y planteamientos de solución presentados por niñas y niños frente a las problemáticas propias del contexto, infantes quienes desde su pensar y accionar postulan ejercicios que favorecen no sólo su realidad sino la de la comunidad en general, hecho que genera en los adultos una visión amplia en torno a la escucha de los postulados realizados por niñas y niños.

\section{Referencias}

Alcaldía municipal de Soacha. (2018). Decreto 236. Recuperado de http://www.alcaldiasoacha.gov.co/secretaria/secretaria-dehacienda/acuerdo/file/32759-decreto- 236 de 13 de agosto de 2018

Aubert, A.; Flecha, A.; García, C.; Flecha, R.; Racionero, S. (2008). Aprendizaje dialógico en la Sociedad de la Información. Barcelona: Hipatia Editorial.

Aubert, A.; Gracia, C. y Racionero, S. (2009). El Aprendizaje dialógico. Cultura y Educación. 21(2), 129-139. 
Bustelo Graffigna, E. (2012). Notas sobre infancia y teoría: un enfoque latinoamericano. Salud colectiva, 8(3), 287-298.

Calvo Muñoz, C. (2012). Del mapa escolar al territorio educativo: Disoñando la escuela desde la educación. Chile: Editorial Universidad de la Serena

Camara, A. N., Morell, I. A., Berñe, A. L., \& Bernet, J. T. (2013). El concepto de ciudadanía construido por jóvenes que vivieron experiencias de participación infantil. Bordón. Revista de pedagogía, 65(3), 93-108.

Cámara, N., \& María, A. (2012). La participación infantil: concepto dimensional en pro de la autonomía ciudadana. Teoría de la Educación. Educación y Cultura en la Sociedad de la Información, 13(2), 380-403.

Caride, J. (2014). La Descolonización Del Niño. Educación Social. Revista de Intervención Socioeducativa, 57(1), 177-179.

Caride, J.; Gradaillé, R.; Caballo, M. (2015). De la pedagogía Social como educación a la educación social como pedagogía. Perfiles Educativos,37(148), 4-11.

Casas, F. (2006). Infancia y representaciones sociales, Childhood and social representations. Política y Sociedad, 43(1), 27-42.

CLACSO (julio de 2000). Consejo Latinoamericano de Ciencias Sociales. Recuperado de https://www.clacso.org/

Contreras, C.; Pérez, A. (2011). Participación invisible: niñez y prácticas participativas emergentes. Revista Latinoamericana de Ciencias Sociales, Niñez $y$ Juventud.9(2), 811-825. ISSN 1692-715X.

Derechos del Niño.(1991). Ley 12 de enero de 1991. Recuperado de https://

www.oas.org/dil/esp/Convencion_Internacional_de_los_De rechos_del_Nino_Colombia.pdf.

Echavarría Grajales, C. (2003). La escuela: un escenario de formación y socialización para la construcción de identidad moral. Revista Latinoamericana de Ciencias Sociales, Niñez $y$ Juventud, 1(2), 15-43. Recuperado de http://www.scielo.org.co/scielo.php? 
script $=$ sci_arttext\&pid=S1692-

$715 X 2003000200006 \& \operatorname{lng}=$ en\&tlng=es.

Escobar, A. (2012), Más allá del tercer mundo: globalización y diferencia, Bogotá, Instituto Colombiano de Antropología e Historia ICANH.

Estrada, M. V.; Madrid-Malo, E.; Gil, L. M. (2000). La participación está en juego. Programa Nacional de Auto Evaluación, Fortalecimiento y Estándares de Instituciones de Protección la niñez. Recuperado de https://www.unicef.org/colombia/informes/la-participacionesta-en-juego

Flecha, R. (1997). Compartiendo palabras: el aprendizaje de las personas adultas a través del diálogo. Barcelona: Paidós.

Francoise, C.R. (2010). Trabajo infantil indígena en Colombia.Organización Internacional del Trabajo, 1 (1), 104

Freire, P. (1997). La Educación como práctica de libertad, Brasil: Siglo XXI.

Gaitán, L. (2006). Sociología de las Infancias, Nueva perspectiva. Madrid: Editorial Síntesis

Gomez, N. M. (2019). La participación infantil como estrategia social para la transformación comunitaria en el Municipio de Soacha, (Doctoral Dissertation, Corporación Universitaria Minuto de Dios, Uniminuto)

Hart, R. (1997). Children's Participation: $\quad e$ eory and Practice of Involving Young citizens in community development and environmental care. Estados Unidos: Routledge

Lambert, C. (2006). Edmund Husserl: la idea de la fenomenología. Teología y vida, 47(4), 517- 529. https://dx.doi.org/10.4067/S0049-34492006000300008.

Maneiro, R. (2011). Un recorrido por el significante Infancia. Perspectivas en Psicología: Revista de Psicología y Ciencias Afines, 8 (2), 95-100. 
Muñoz, P.; Gamboa A., y Montes A., (2015). Participación crítica y democrática: comprensión de los discursos de actores educativos. Revista Zona Próxima, 22(1), 56-68

Nemocón, J. (2014). Política Pública de primera infancia, infancia y adolescencia. Alcaldía de Soacha. Recuperado de http://alcaldiasoacha.gov.co/atencion-a-laciudadania/notificacionesporaviso?download $=1552$ :acuerdo-24-de-2015\&start $=90$

Panotto, N. (Julio-diciembre, 2014). Pentecostalismos y construcción de identidades sociopolíticas. Desafios, 26(2), 73-96.

Pavez Soto Iskra (2012) Sociología de la Infancia: las niñas y niños como actores sociales. Revista de Sociología, 27(1), 81-102

Peña, N. C. (2017). Los niños y las niñas, ¿sujetos políticos?: construcciones posibles desde la escuela y el aula. Dialnet, $16(2), 228-241$.

Quijano, A. (s.f.). Colonialidad del poder, eurocentrismo y América Latina. En libro: La colonialidad del saber: eurocentrismo y ciencias sociales. Buenos Aires, Argentina: CLACSO

Racionero, S; Ortega, S; García, R; Flecha, R. (2012). Aprendiendo contigo. Barcelona, España: Hipatia Editorial. ISBN: 97884-938226-3-7.

Racionero, S; Padrós, M. (2010). e Dialogic Turn in Educational Psychology. Journal of Psychodidactics, 15(2), 143-162

Redondo, G. (2015). Liderazgo dialógico en Comunidades de aprendizaje, University of Cambridge. Revista Omnia Science, 11(3), 437-457.

Ruíz Corbella, M.; Martín Cuadrado, A.; Cano Ramos , M. (2015). La consolidación del perfil profesional del educador social. Perfiles Educativos, 37(148), 12-19

Salazar Asencio, J. (2015). Pedagogía Social y educación social: reflexiones y experiencia. Perfiles Educativos, 37(148), 2-3.

Sanabria, M. (2015). Mántica de la palabra. Bogotá: Universidad Pedagógica Nacional. 
Siu Lin, S. (2015). La participación de la infancia desde la Infancia. La Construcción de la Participación Infantil a Partir del Análisis de los Discursos de Niños y Niñas (Tesis Doctoral). Segovia, España: Universidad de Valladolid Facultad de Educación y Trabajo Social.

Revista Mexicana De Ciencias Políticas Y Sociales, 41.

Tonucci, F. (2015) .La Ciudad de los niños. Barcelona España: Ed. Grao.

Trilla Bernet, J., \& Novella Cámara, A. N. (2011). Participación, democracia y formación para la ciudadanía. Los consejos de infancia. Revista de educación,356(1), 23-43.

Unicef (1990). Convención de los Derechos del niño. Plan de Acción de la Cumbre Mundial a favor de la Infancia. Recuperado de https://www.unicef.es/publicacion/convencion-sobre-losderechos-del-nino

UNICEF. (2003). La participación de los niños: mitos y realidades. Recuperado de https://www.unicef.org/spanish/sowc03/contents/pdf/chapte rs.pdf

UNICEF. (2005).Convención sobre los derechos de los niños y las niñas. https://www.unicef.es/causas/derechos-ninos/convencionderechos-ninos 